Meta

Journal des traducteurs

Translators' Journal

\title{
Los nombres propios y su tratamiento en traducción
}

\section{Carmen Cuéllar Lázaro}

Volume 59, numéro 2, août 2014

URI : https://id.erudit.org/iderudit/1027480ar

DOI : https://doi.org/10.7202/1027480ar

Aller au sommaire du numéro

\section{Éditeur(s)}

Les Presses de l’Université de Montréal

ISSN

0026-0452 (imprimé)

1492-1421 (numérique)

Découvrir la revue

Citer cet article

Cuéllar Lázaro, C. (2014). Los nombres propios y su tratamiento en traducción. Meta, 59(2), 360-379. https://doi.org/10.7202/1027480ar

\section{Résumé de l'article}

La présente étude vise à analyser les stratégies employées par différents traducteurs pour la traduction des noms propres dans les oeuvres littéraires. Sur la base de trois oeuvres de la littérature contemporaine en langue allemande, on analysera les traductions paires espagnoles de chaque traducteur, ce qui nous permettra, tout d'abord, de mettre en lumière les techniques utilisées dans la traduction des noms propres et leurs résultats, puis d'extrapoler à l'ensemble des trois doublets, pour voir s'il y a des aspects diachroniques qui distinguent les deuxièmes traductions (TM2) des premières traductions (TM1), c'est-à-dire s'il y a une relation entre la TM1 et TM2 analysées. L'analyse de la méthode employée par les six traducteurs est précédée d'une réflexion qui fournit des réponses à plusieurs questions liées aux propriétés idiosyncrasiques des noms propres et au traitement spécial en traduction
Ce document est protégé par la loi sur le droit d'auteur. L'utilisation des services d’Érudit (y compris la reproduction) est assujettie à sa politique d'utilisation que vous pouvez consulter en ligne.

https://apropos.erudit.org/fr/usagers/politique-dutilisation/ 


\title{
ÉTUDES ET PROSPECTIVES
}

\section{Los nombres propios y su tratamiento en traducción}

\author{
CARMEN CUÉLLAR LÁZARO \\ Universidad de Valladolid, Valladolid, España \\ carmen.cuellar@lesp.uva.es
}

\begin{abstract}
RÉSUMÉ
La présente étude vise à analyser les stratégies employées par différents traducteurs pour la traduction des noms propres dans les œuvres littéraires. Sur la base de trois œuvres de la littérature contemporaine en langue allemande, on analysera les traductions paires espagnoles de chaque traducteur, ce qui nous permettra, tout d'abord, de mettre en lumière les techniques utilisées dans la traduction des noms propres et leurs résultats, puis d'extrapoler à l'ensemble des trois doublets, pour voir s'il y a des aspects diachroniques qui distinguent les deuxièmes traductions (TM2) des premières traductions (TM1), c'est-à-dire s'il y a une relation entre la TM1 et TM2 analysées. L'analyse de la méthode employée par les six traducteurs est précédée d'une réflexion qui fournit des réponses à plusieurs questions liées aux propriétés idiosyncrasiques des noms propres et au traitement spécial en traduction.
\end{abstract}

\section{ABSTRACT}

The present study aims to analyze the strategies employed by different translators for the translation of proper names in literary works. Based on three works of contemporary literature in German, it will analyze the double Spanish translations of each, allowing us to understand, firstly, the techniques used in the translation of proper names by translators, each of the translation doublets, and then to extrapolate the results to all three doublets, to see if there are any diachronic aspects which distinguish the second translations (TM2) from the first translations (TM1), that is, if there is any relationship between the TM1 and TM2 analyzed. The analysis of the behavior of the six translators is preceded by a reflection which provides answers to several questions related to the idiosyncratic properties of proper names and special treatment in Translation.

\section{MOTS-CLÉS/ KEYWORDS}

nom propre, traduction littéraire, référence culturelle, anthroponyme, toponyme proper name, literary translation, cultural reference, anthroponym, toponym

\section{Acercamiento al estudio del nombre propio: la onomástica}

Las propiedades idiosincrásicas del nombre propio hacen que sea un elemento especialmente interesante para su análisis, ya sea desde un enfoque intralingüístico como también interlingüístico, es decir, en el contexto de su traducción a otra lengua. Cuando nos acercamos a su estudio surgen varias cuestiones que vamos a ir analizando a lo largo de este trabajo. 
Se denomina onomástica la ciencia que trata la catalogación y el estudio de los nombres propios. Tras esta definición se plantea la primera cuestión al preguntarnos qué estatus lingüístico tiene la onomástica. Para reflexionar sobre el tema traemos a estas páginas las investigaciones de un clásico en el análisis de los nombres propios en el contexto francófono, Jean Molino, quien señala que estos han tenido un lugar importante en disciplinas como la antropología o la lógica, sin embargo, hasta épocas recientes el nombre propio «est bien un parent pauvre de la linguistique» (Molino 1982: 5). Más recientemente, Jean-Louis Vaxelaire se expresa también en estos términos, destacando que hasta los años setenta «[n]ous verrons que rares sont les linguistes à s'intéresser à ce sujet» (Vaxelaire 2005: 17)1.

No obstante, la distinción entre nombre propio y común se remonta, sin embargo, hasta los orígenes de la gramática occidental, es decir, a las gramáticas clásicas grecolatinas. En este sentido, Donato (s. IV d. C.) ya distinguía entre unius nomen [nombre de uno solo], frente al multorum nomen [nombre de varios]. El nombre propio era considerado como onoma kúrion, traducido del griego al latín por nomen proprium, el nombre que verdaderamente nombra, el verdadero nombre, el que designa seres individuales. Sin embargo, una vez hecha esta distinción, no puede decirse que el nombre propio haya interesado especialmente al lingüista. Con el nacimiento de la lingüística histórica y comparada se constituyó una disciplina auxiliar (limítrofe y marginal) en la que el estudio de los nombres propios iba a tener una vida independiente: la onomástica, centrada en la investigación del origen de los nombres propios, especialmente los nombres de personas y lugares. Sin embargo, la onomástica se mantuvo al margen dentro de las corrientes estructuralista y generativista. De hecho, en el siglo XX, fueron lógicos y filósofos quienes dedicaron especial atención al nombre propio y posteriormente sus aportaciones despertaron el interés de los lingüistas, a finales de la década de los setenta, atraídos por los aspectos semántico-referenciales del mismo (Molino 1982; Fernández Leborans 1999; Grass 2002).

L'exemple de Lévi-Strauss montre que le sujet du nom propre a été abordé au $\mathrm{XX}^{\mathrm{e}}$ siècle par d'autres disciplines que la linguistique et la logique. Les ethnologues ou les critiques littéraires ont rédigé une littérature abondante sur le nom propre. Il serait par conséquent possible de consacrer plusieurs volumes à ce sujet. (Vaxelaire 2005: 591)

En esta línea, también se ha puesto de relieve el carácter interdisciplinar de los estudios onomásticos por otros investigadores, como el lingüista alemán Ernst Eichler, quien destaca su conexión con otras disciplinas, entre ellas, la arqueología y la geografía: «die interdisziplinären Verbindungen zu historischen Disziplinen (vor allem zur Archäologie, Siedlungsgeschichte und Geographie usw)» (Eichler 1995: 1). Este autor defiende a la vez su emancipación como disciplina lingüística, en base al interés creciente de los estudios onomásticos desde esa perspectiva: «Dies garantiert ihr den Status einer wichtigen linguistischen Disziplin» (Eichler 1995: 7; véase también Pulgram 1954; Sorensen 1963; Zabeeh 1968; Morala 1986; Allerton 1987; GaryPrieur 1991; Molino 1982; Folkart 1986; Vaxelaire 2005).

Enlazando con el estatus lingüístico de la onomástica, nos cuestionamos cómo definir un nombre propio y nos acercamos a su definición a través de las distintas taxonomías en las categorías que pueden reunirse bajo este término. Las clasificaciones varían de unos gramáticos a otros, dejando algunos investigadores incluso la lista 
abierta para aquellos nombres de designación ocasional, es decir, cualquier objeto o entidad puede ser, en una circunstancia concreta, un nombre propio (véase Molino 1982; Gläser 1976; Vaxelaire 2005).

Un acercamiento epistemológico al estudio del nombre propio debe tener en cuenta esta multiplicidad, así como también la heterogeneidad interior de cada una de las categorías. Esta diversidad es lo que le lleva a afirmar a Molino que no se puede dar una definición «simple et cohérente» del nombre propio; ni el criterio ortográfico, ni el sintáctico, ni el referencial, ni el semántico permiten delimitar, sin ambigüedad, el campo de los nombres propios del de los comunes (Molino 1982: 6; Grass 2002: 7; Vaxelaire 2005: 71-149). El Diccionario de la Real Academia en su vigésima primera edición (1992) hacía una distinción sobre bases semánticas y lo definía como:

El que se aplica a seres animados o inanimados para designarlos y diferenciarlos de otros de su misma clase, y que, por no evocar necesariamente propiedades de dichos seres, puede imponerse a más de uno (Antonio, Toledo), incluso a seres de distinta clase (Marte). (Diccionario de la Real Academia 1992: 1445) ${ }^{2}$

En la edición posterior de 2001, se modifica la definición precisamente sobre esa base: «El que, sin tener rasgos semánticos inherentes, se aplica a seres animados o inanimados para designarlos; p. ej., Antonio, Toledo» (Diccionario de la Real Academia 2001: 1587) ${ }^{3}$, manteniéndose en los mismos términos en el avance de la vigésima tercera edición, en su versión online ${ }^{4}$.

Esta variación en la definición hace que nos planteemos si los nombres propios tienen un contenido semántico. La controversia suscitada sobre la posible semántica de los nombres propios ha interesado no sólo a lingüistas, sino también a filósofos y a teóricos de la traducción, trayendo consigo posturas totalmente opuestas ${ }^{5}$. Por un lado, hay autores que consideran que estos no tienen significado, no son connotativos, sino que simplemente denotan a los individuos que designan. Entre ellos destaca el filósofo inglés John Stuart Mill, quien en su obra A System of Logic defiende la postura de que los nombres propios no tienen significado, solamente identifican, son «unmeaning mark» (Mill 1875: 37). En esta misma línea, Otto Funke señala que son etiquetas que marcan a los individuos como el tejuelo de un libro en una biblioteca: «Etiketten oder Ordnungszeichen, die gedanklich den Individuen zum Unterschied von anderen anhaften wie die Büchersignaturen in einer Bibliothek» (Funke 1925: 77) [Etiquetas o marcas que hacen referencia internamente a los individuos diferenciándolos de otros como las signaturas de los libros en una biblioteca]. También Dietrich Gerhardt defiende esa postura al definir los nombres propios como «bloßes diakritisches Zeichen» [simples signos diacríticos] con «semantische Bedürfnislosigkeit» [austeridad semántica] (Gerhardt 1949/1950: 7 y 5).

Para otros investigadores, sin embargo, los nombres propios poseen incluso una semántica más rica que los comunes, como postula el lingüista y filósofo danés Otto Jespersen: "proper names [...] 'connote' the greatest number of attributes» (1924/1992: 66). De la misma manera, el lingüista austríaco Ernst Pulgram los considera «the most meaningful of all nouns» (1954: 187). Antes que ambos, el también lingüista, en este caso francés, Michel Bréal ya se había expresado con estas palabras: «La différence avec les noms communs est une différence tout intellectuelle. Si l'on classait les noms d'après la quantité d'idées, ils sont les plus significatifs de tous, étant les plus individuels» (Bréal 1924/1976: 183). 
Esta controversia sobre su semanticidad ha sido un tema manido en la literatura científica de las dos últimas décadas. Desde un enfoque traductológico, parece evidente que la carga semántica que pueda poseer un nombre propio de la ficción literaria, su grado de connotación, estarán marcados por la intencionalidad del escritor y por ende influirán en la decisión del traductor, como autor de la re-enunciación de ese texto.

L'onomastique constitue l'un des lieux où la ré-énonciation se fait sentir avec les plus d'acuité. Aussi banal soit-il, le nom propre est, ou devient grâce à la traduction, porteur de sens, soit qu'il connote à travers son signifiant, soit qu'il y ait adéquation de son dénoté au personnage qu'il désigne. (Folkart 1991: 136)

Enlazando con esta idea, el nombre propio es, o se hace gracias a la traducción, portador de sentido y, en este contexto, parece paradójico cuestionarse si los nombres propios son traducibles, lo que nos introduce a su vez en el próximo capítulo.

\section{Sobre la traducibilidad del nombre propio}

Desde una perspectiva lingüística, algunos autores consideran traducción solo la traducción stricto sensu, es decir, el caso de nombres propios semánticamente transparentes: «Übersetzbar ist nur das charakterisierende Element der lexikalischen Bedeutung; wo dies nicht ein gewisses Mindestmaß erreicht, kann von Übersetzung keine Rede sein» (Fleischer 1964: 372). Desde este enfoque, aquellos que tienen un contenido semántico son traducibles y, por el contrario, los que no están semantizados, no pueden traducirse (Störig 1963; Fleischer 1964; Neubert 1973; Gläser 1976; Gutschmidt 1980; Zimmer 1981; Probst 1984; Torre 1994). El lingüista polaco Witold Mańczak define al nombre propio precisamente por su intraducibilidad:

[...] parmi toutes les définitions en question, la meilleure est celle d'après laquelle la différence entre le nom commun et le nom propre consiste en ce que celui-ci, dans la presque totalité des cas, ne se traduit pas dans les langues étrangères. (Mańczak 1969: 290)

Bajo esta perspectiva, aquellos sin carga semántica no pueden ser traducidos, sin embargo, pueden ser adaptados mediante asimilaciones formales (fonéticas, fonológicas, ortográficas) al propio sistema lingüístico de la lengua meta (LM) (véase la diferenciación entre «Namenvarianten» y «Namendubletten» en Fleischer (1973a: 6-10 1973b: 53-62 y Kalverkämper (1995: 1019-1020).

Figuras señeras, como Valentín García Yebra, tampoco consideran procedimientos de traducción propiamente dichos la transferencia, lo que este investigador denomina «préstamo inadaptado» o extranjerismo, ni la adaptación, es decir, el «préstamo naturalizado» (García Yebra 1989: 335-340). En la misma línea se expresa Jean Delisle, para quien «[l]'emprunt est un cas de non traduction» (Delisle 1984: 88).

El término traducción es empleado por otros autores para referirse también a la adaptación gráfica: «Einige Vornamen sind insofern übersetzt, als sie graphisch angepaßt werden» (Zimmer 1981: 101). Peter Newmark sostiene en su obra Approaches to Translation (1988b) que al carecer los nombres propios de carácter lingüístico no pertenecen a los diccionarios sino a las enciclopedias y son intraducibles:

In theory, names of single persons or objects are 'outside' languages, belong, if at all, to the encyclopaedia not the dictionary, have, as Mill stated, no meaning or connotations, are, therefore, both untranslatable and not to be translated. (Newmark 1988b: 70) 
Esta afirmación es matizada por Newmark en la exposición de los procedimientos de traducción, incluyendo dentro de la "transferencia" (es decir, préstamo) la mayor parte de los nombres de personas y los nombres geográficos sin traducción reconocida (1988a: 82-83). Además dedica dos apartados a esta problemática bajo el título: “The translation of proper names" (1988a: 35-36; 214-216). El carácter normativo de su obra, de gran influencia en didáctica de la traducción no sólo en Gran Bretaña sino también en España, se refleja asimismo en lo referente a los nombres propios.

Con respecto a los antropónimos, la presencia o ausencia de connotación marca la diferencia en el proceder a la hora de ser traducidos. Cuando los nombres de pila y apellidos no tienen connotaciones en el texto, se suelen transferir, con lo cual se mantiene su nacionalidad, aunque exceptúa algunos casos: los nombres de papas, monarcas, príncipes y santos, etc. En cambio, para los nombres de la literatura que tienen connotaciones hace la siguiente propuesta:

Where both connotations (rendered through sound effects and/or transparent names) and nationality are significant, I have suggested that the best method is first to translate the word that underlies the SL proper name into the TL, and then to naturalise the translated word back into a new SL proper name - but normally only when the character's name is not yet current amongst an educated TL readership. (Newmark 1988a: 215)

En cuanto a los términos geográficos, sostiene, con ese carácter prescriptivo que caracteriza su obra, que deben consultarse los atlas o diccionarios geográficos más recientes, tener en cuenta la versión oficial de los mismos y no inventarse términos nuevos (Newmark 1988a: 216).

En este sentido, la idea de que los nombres propios son intraducibles se ha extendido tradicionalmente en traductología, poniéndose de manifiesto en el expresivo título de la obra de Thierry Grass (2002): Quoi! Vous voulez traduire «Goethe»? Sin embargo, tal y como señala Vaxelaire, creer en su intraducibilidad supone no percibir la heterogeneidad de las distintas categorías que lo conforman, olvidarse de la diversidad de las prácticas traductológicas a lo largo de épocas, lenguas y países distintos, así como obviar la heterogeneidad según los géneros textuales, en definitiva, en la interacción entre todos estos elementos (Vaxelaire 2011: 27; 2005: 99-101). Siguiendo este argumento, será imprescindible que el traductor tenga en cuenta la función del nombre propio en el texto en el que se enmarca y el lector al que va dirigido (Neubert 1973; Hellfritzsch 1973; Gläser 1976; Molino 1982; Probst 1984; Ballard 2001; Nord 2003; Lungu-Badea 2011; Ballard 2011: 45).

De este modo, en los últimos años han surgido nuevos estudios que destacan, a través del análisis de distintas técnicas traductológicas, que estos no están exentos del proceso de traducción. Bajo la dirección de T. Grass, J. Humbley y J. L. Vaxelaire, se publican en la revista Meta en 2006 una serie de trabajos de gran interés por el enfoque multilingüe que se ofrece en el tratamiento de los nombres propios. Uno de los objetivos de estos estudios es el de sentar las bases para su procesamiento automático, teniendo como base la traducción multilingüe de los nombres propios en el proyecto PROLEX (Grass, Humbley y Vaxelaire 2006).

En 2011, la revista Translationes publica asimismo un sugerente monográfico que aborda la problemática sobre la traducibilidad de los nombres propios, ofreciendo una imagen global del tema a partir de una serie de artículos realizados desde distintos enfoques (teóricos y empíricos, normativos y descriptivos) (Lungu Badea y Pelea 2011). 
Desde esta nueva perspectiva, desde una visión global del proceso traductivo, defendemos la traducibilidad del nombre propio, al considerar la traducción como proceso (no como fin) y el uso de distintas técnicas, incluidas la repetición y la adaptación, lleva consigo la reflexión y la decisión del traductor de emplear un determinado procedimiento en su traducción (véase Moya 2000; Franco Aixelá 2000; Ballard 2001; Grass 2002; Nord 2003; Vaxelaire 2011). Esto se pone de manifiesto en el siguiente apartado en el que se va a analizar el proceder de los distintos traductores.

\section{Análisis de la traducción de nombres propios en dobletes literarios}

\subsection{Consideraciones preliminares}

A continuación se lleva a cabo un análisis de traducción del nombre propio basado en la comparación de dos versiones diferentes al español de una misma obra literaria en lengua alemana, realizada por dos traductores distintos, lo que denominamos doblete de traducción. En concreto analizamos la novela del suizo Friedrich Dürrenmatt Der Richter und sein Henker $(1952)^{6}$, algunos relatos de Hermann Hesse publicados en 1945 con el título Traumfährte (Hesse 1945/1987) ${ }^{7}$, y relatos de Heinrich Böll que se reúnen en un libro titulado Erzählungen, Hörspiele, Aufsätze, publicado en $1962^{8}$. Los tres dobletes elegidos para el estudio pertenecen al género de la prosa, y los criterios para su elección han sido el prestigio internacional del escritor y una diferencia cronológica entre las primeras y las segundas traducciones de más de 15 años. 27 años separan las dos traducciones de la novela de Dürrenmatt; entre el texto meta primero (TM1) y el texto meta segundo (TM2) de los relatos de Hesse hay una distancia cronológica de 26 años; por último, las dos versiones de los relatos de Böll han sido publicadas con un intervalo de tiempo de 17 años. Desde un enfoque temporal, esta diferencia nos permite incorporar una perspectiva diacrónica en el estudio.

La metodología empleada en este trabajo se inscribe dentro del enfoque descriptivo. El objetivo del análisis será observar las estrategias empleadas por los traductores para la traducción de los nombres propios en las obras literarias mencionadas. Nos vamos a centrar en el análisis descriptivo de los dos tipos de nombres propios por excelencia, los antropónimos y los topónimos, entendiendo esos últimos en su sentido más amplio (Ballard 2001).

Partiendo de la hipótesis de que la repetición suele ser la técnica más habitual en el caso de la traducción de los nombres propios, a través de este estudio conoceremos, en primer lugar, las técnicas más empleadas por los traductores en cada doblete y posteriormente se extrapolarán los resultados al conjunto de los tres dobletes, para ver si desde una perspectiva diacrónica hay alguna relación entre los TM1 y los TM2 analizados, es decir, si hay aspectos que caractericen a las segundas traducciones con respecto a las primeras.

El estudio comparativo de este tipo de corpus tiene múltiples ventajas, como indica Franco Aixelá (2000:144,138), por el carácter descriptivo y no prescriptivo del mismo, con el consiguiente avance en el carácter científico y no especulativo de la traductología; además, este tipo de análisis nos permite observar cómo han resuelto los traductores los problemas traductológicos a los que se han enfrentado, avanzando así en el conocimiento de la realidad de la práctica traductora. Como consecuencia de ello, el campo de la didáctica de la traducción se ve enriquecido con propuestas de estrategias de traducción basadas en la realidad del traductor profesional. 
La taxonomía de la técnicas traslatorias que hemos encontrado a lo largo del análisis, teniendo en cuenta una escala que va desde una mayor conservación del universo cultural del texto original (TO) hasta su sustitución por el universo cultural del texto meta (TM), u omisión, es la siguiente: repetición, adaptación, traducción lingüística, glosa intratextual, glosa extratextual, neutralización, naturalización y omisión (Moya 2000: 173-182; Franco Aixelá 2000: 84-94).

\subsection{Resultados del análisis de los nombres propios traducidos}

En el doblete de la novela de Friedrich Dürrenmatt se han observado estrategias distintas, por parte de los traductores, a la hora de trasladar los nombres propios (104 en total). En ambos casos, la repetición es la técnica más empleada, sobre todo en el TM2 (Dürrenmatt 1952/1989, traducido por Del Solar) ${ }^{9}$, con el 76\% frente al 49\% en el TM1 (Dürrenmatt 1952/1970, traducido por De Luque) ${ }^{10}$. Teniendo en cuenta que la repetición es la técnica más conservadora, se podría generalizar diciendo que el primer traductor intenta, mediante la adaptación (28\%) y traducción lingüística (19\%), acercar el texto alemán a los lectores, dando prioridad a la inteligibilidad y evitando el efecto de extrañeza (véase la dicotomía domestication vs. foreignization en Venuti 1995: 19-20 y Venuti 1998: 24; una reflexión sobre estos términos desde una nueva perspectiva del concepto visibility en Folkart 2007: 280-283). En la segunda traducción, por el contrario, el alto porcentaje en la técnica de la repetición muestra que se emplea menos la adaptación (10\%) y la traducción lingüística (11\%) con lo que se pretende que sea el lector el que se dirija hacia el original. Precisamente las divergencias que se dan entre los traductores, que representan el 34\% del total, vienen motivadas por la disposición del primer traductor a la castellanización de los nombres propios, bien sean antropónimos o topónimos, mediante adaptación terminológica u ortográfica: Lignières (TO, TM2) vs. Ligniere (TM1), Prêles (TO, TM2) vs. Preles (TM1), Gümmenen (TO, TM2) vs. Gummenen (TM1), Schüpfen (TO, TM2) vs. Schupfen (TM1), Frankfurt am Main (TO, TM2) vs. Francfort del Meno (TM1), Anna (TO, TM2) vs. Ana (TM1), Oskar (TO, TM2) vs. Oscar (TM1), Niklaus Manuel (TO, TM2) vs. Nicolás Manuel (TM1), Alphons Clenin (TO, TM2) vs. Alfonso Clenin (TM1) y Ulrich Schmied (TO, TM2) vs. Ulrico Schmied (TM1).

Siguiendo con la tendencia a acercar el texto a los lectores, el primer traductor opta por la traducción lingüística en el caso de nombres propios de lugares: Kasinoplatz (TO, TM2) vs. plaza del Casino (TM1), Bantigerstraße (TO, TM2) vs. calle Bantiger (TM1), Altenbergstraße (TO, TM2) vs. calle Altenberg (TM1) y Bärenplatz (TO, TM2) vs. plaza del Oso (TM1).

(1) Noch am selben Abend ging Bärlach zu seinem Arzt am $\underline{B a ̈ r e n p l a t z}$, Doktor Samuel Hungertobel.

(Dürrenmatt 1952/1986: 82)

(a) Aquella misma noche Bärlach visitó a su médico, el doctor Samuel Hungertobel, que vivía en la plaza del Oso.

(Dürrenmatt 1952/1970: 107, traducido por De Luque [1962])

(b) Aquella misma tarde fue Bärlach a ver a su médico, el doctor Samuel Hungertobel, en la Bärenplatz.

(Dürrenmatt 1952/1989: 127, traducido por Del Solar) 
El sustantivo Bären aparece también al principio de la novela como nombre de un hotel y en este caso el traductor del TM1 opta por la adaptación ortográfica: Hotel Bären (TO), hotel Bären (TM2,) vs. hotel Baren (TM1).

El hagiotopónimo St. Petersinsel es empleado por Dürrenmatt en su obra como Peterinsel y el traductor del TM1, en su idea de acercamiento del TO al lector, realiza en este nombre propio poliléxico, traducción lingüística y adaptación, como se observa en su contexto:

(2) Bärlach öffnete das Fenster und blickte auf den See hinunter. Über der Peterinsel standen einige Sterne.

(Dürrenmatt 1952/1986: 25)

(a) Bärlach abrió la ventanilla y miró el lago, allá abajo. Por encima de la isla de Pedro había algunas estrellas.

(Dürrenmatt 1952/1970: 34, traducido por De Luque [1962])

(b) Bärlach abrió la ventanilla y miró el lago, allá abajo. Por encima de la isla de Peter brillaban algunas estrellas.

(Dürrenmatt 1952/1989: 38, traducido por Del Solar)

En lo que respecta al nombre compuesto Waisenhausplatz es vertido erróneamente al castellano por el primer traductor como patio del orfanato, en minúscula, perdiendo la caracterización de nombre propio y la connotación del TO. El segundo traductor, sin embargo, acertadamente, lo conserva:

(3) Dann wandte er Bärlach den Rücken zu und schaute zum Fenster auf den Waisenhausplatz hinaus, der voller Kinder war.

(Dürrenmatt 1952/1986: 13)

(a) A continuación, Lutz se puso a mirar por la ventana al patio del orfanato, que estaba lleno de niños.

(Dürrenmatt 1952/1970: 18, traducido por De Luque [1962])

(b) Luego volvió la espalda a Bärlach y se puso a mirar por la ventana hacia la Waisenhausplatz, abarrotada de niños.

(Dürrenmatt 1952/1989: 21, traducido por Del Solar)

Coinciden ambos traductores en introducir una glosa intratextual al verter los nombres propios Twanner y Ligerzer, fruto de la derivación deonomástica de los topónimos suizos Twann y Ligerz:

(4) Der neue Twanner schien Bärlach nicht gutgetan zu haben, [...].

(Dürrenmatt 1952/1986: 15)

(a) El vino nuevo de Twann no pareció haberle sentado a Bärlach, [...].

(Dürrenmatt 1952/1970: 21, traducido por De Luque [1962])

(b) El vino nuevo de Twann no pareció sentarle bien a Bärlach, [...]

(Dürrenmatt 1952/1989: 25, traducido por Del Solar) 
(5) „Was wollen wir trinken?« sagte Bärlach, »Ligerzer? «.

(Dürrenmatt 1952/1986: 103)

(a) ¿Qué beberemos? -dijo Bärlach-. ¿Vino de Ligerz?

(Dürrenmatt 1952/1970: 138, traducido por De Luque [1962])

(b) ¿Qué bebemos? -preguntó Bärlach-. ¿Vino de Ligerz?

(Dürrenmatt 1952/1989: 158, traducido por Del Solar)

En el doblete de los relatos de Hermann Hesse (98 nombres propios en total) también en el TM2 (Hesse 1945/1976, traducido por Bofill) ${ }^{11}$ el porcentaje más elevado es para la técnica de la repetición (71\%), seguido de la adaptación (19\%), y la traducción lingüística (5\%). En el TM1 (Hesse 1945/1950, traducido por Pintó) ${ }^{12}$, la repetición desciende hasta el 59\%, en favor de la adaptación (29\%) y la traducción lingüística (8\%). Las divergencias entre las traducciones ascienden al 34\% y también como en el caso de Dürrenmatt, el primer traductor recurre a una mayor modificación del nombre propio del TO, no obstante, el cambio que sufre no siempre está motivado por el deseo de dar prioridad a la cercanía del texto por parte de los lectores en lengua española, como veíamos en el caso del autor suizo.

De esta manera, en el relato Schwäbische Parodie (Hesse 1945/1987) los apellidos Fischer y Bohnenberger, semánticamente transparentes, se convierten, por un grave error, en "pescadores y recolectores de judías" en el TM1. Como es sabido, el criterio ortográfico de la mayúscula, definitorio del nombre propio en algunas lenguas, no es compartido por el alemán, que identifica al nombre, ya sea propio o común, con la grafía mayúscula:

(6) Erwähnt sei nur, daß die wunderlichen Ausdrücke 'knorzen' und 'Knorzer' nach den genialen Forschungen Fischers und Bohnenbergers zweifellos jenem Sagenkreise entstammen; [...].

(Hesse 1945/1987: 440)

(a) Digamos aún que ciertas expresiones, según las geniales investigaciones de pescadores y recolectores de judías, tienen su origen en este ciclo de leyendas, [...].

(Hesse 1945/1950: 112, traducido por Pintó)

(b) Sólo señalaré aquí que, según las geniales investigaciones realizadas por Fischer y Bohnenberger, las maravillosas expresiones 'knorzen' y 'Knorzer' derivan sin duda alguna de dicho grupo de leyendas; [...].

(Hesse 1945/1976: 135, traducido por Bofill)

Algunas de las variaciones en los nombres propios de los relatos de Hesse pueden deberse a despistes o errores tipográficos: en el relato Vogel, el antropónimo Schuster (TO, TM2) se convierte en Sehuster (TM1) ${ }^{13}$; en Schwäbische Parodie, Justinus Kerner (TO, TM2) es Instinus Kerner (TM1), y en Vom Steppenwolf, Bopfingen (TO, TM2) pasa a Boptingen (TM1).

Un intento de acercamiento al lector puede verse en el cambio de Martin Kurtz (Schwäbische Parodie [TO] y TM2) por Martín Kurz (TM1), es decir, castellanización del nombre de pila con la tilde gráfica y omisión de una consonante en el apellido, así como también en el antropónimo Vishnu (Kindheit des Zauberers [TO] y TM2) que pasa a Visnú (TM1), Shiva (Kindheit des Zauberers [TO] y TM2) a Siva (TM1) y Achilles Zwilling (Schwäbische Parodie [TO]) es Achilles Zwillig (TM2), que el primer traductor simplifica como Aquiles (TM1). 
En la misma línea, suponemos que el siguiente ejemplo, del relato Schwäbische Parodie, es un intento de acercar el texto original al lector por parte del primer traductor, empleando la estrategia de naturalización al sustituir el nombre propio original Zeus, el dios de la mitología griega, por un referente distinto, más cercano al lector, Júpiter, su equivalente en la mitología romana. En el caso del TM2, se introduce una glosa intratextual o explicitación: la palabra ninfa, integrada en el texto término como parte indistinta del mismo.

(7) [...], wenn Hammelehle in seiner sonst verdienstvollen Dissertation «Herzog Eugen der Langhaarige in seinen Beziehungen zum Humanismus» in diesem Bericht lediglich eine humanistisch-klassizistische Nachdichtung des Abenteuers zwischen Zeus und der Europa glaubt erkennen zu dürfen.

(Hesse 1945/1987: 441)

(a) [...], a pesar de que Hammelehle en su, por otra parte, meritoria disertación "El duque Eugenio, el de largos cabellos, y sus relaciones con el humanismo", sólo vea en él un calco clásico humanístico de la fábula de Lúpiter y Europa.

(Hesse 1945/1950: 113, traducido por Pintó)

(b) [...] y en todo caso nos parece precipitada la conclusión de Hammelehle, quien en su disertación, por lo demás meritoria, sobre «El duque Eugen el Velloso y su relación con el humanismo» cree poder identificar en este relato únicamente una versión humanístico-clásica del tema de la aventura de Zeus y la ninfa Europa.

(Hesse 1945/1976: 135, traducido por Bofill)

En este primer traductor se percibe a lo largo de la obra una tendencia relativamente sistemática a adaptar los antropónimos, muy especialmente los nombres de pila que comparten etimología con el español, como ilustra el ejemplo anterior en el que Eugen se convierte en Eugenio. Fenómeno, por otra parte, muy común en las traducciones de los años cincuenta en España (Franco Aixelá 2000: 261). En el caso del epíteto, ambos traductores optan por la traducción lingüística, aunque con distinto resultado e introduciendo diferentes matices para el lector.

Una excepción en el comportamiento del primer traductor supone el siguiente ejemplo, del relato Vogel, en el que se mantiene el nombre propio, dando por hecho el conocimiento del lector de la dinastía Hohenstaufen. No obstante, se observa error en la grafía, algo que, como ya hemos comentado, caracteriza esta traducción: Hoheustaufen ${ }^{14}$. El segundo traductor, sin embargo, emplea una neutralización absoluta, omitiendo el nombre propio y explicitando su significado:

(8) [...], der einst ein Zeuge von Abels Tod oder ein Hohenstaufe oder Prinz oder Zauberer gewesen war [...].

(Hesse 1945/1987: 475)

(a) [...] secular testigo de la muerte de Abel, Hoheustaufen, príncipe, mago, [...]. (Hesse 1945/1950: 150, traducido por Pintó)

(b) [...] ese personaje que había presenciado la muerte de Abel o había pertenecido a una noble familia o había sido un príncipe o un brujo [....].

(Hesse 1945/1976: 176, traducido por Bofill) 
Los traductores de los relatos de Heinrich Böll (1962), por último, tienden a dejar los nombres propios (70 en total) tal y como aparecen en el original, es decir, mediante la técnica de la repetición, el 76\% en el TM1 (Böll 1962/1964, traducido por Fontseré) ${ }^{15}$ y 70\% en el TM2 (Böll 1962/1981, traducido por Moral Arroyo) ${ }^{16}$. En los escasos ejemplos del corpus en que se diferencian las soluciones dadas por los traductores (15\%) - el mayor número de soluciones idénticas entre los TM de cada doblete se da precisamente en las traducciones de los relatos de Böll, con el restante $85 \%$ - es el segundo traductor el que emplea técnicas que acercan o neutralizan todo o parte del exotismo del referente cultural: neutralización absoluta en su club (TM2) frente a Gaffel-Club (Wie in schlechten Romanen [TO], TM1), omitiendo el nombre propio:

(9) Weil ich wei $\beta$, da $\beta$ er im Gaffel-Club sitzt und Schach spielt, wie jeden Mittwochabend um diese Zeit.

(Böll 1962: 118)

(a) Porque sé que está en el Gaffel-Club jugando al ajedrez, como todos los miércoles a estas horas.

(Böll 1962/1964: 190, traducido por Fontseré)

(b) Porque sé que está en su club jugando al ajedrez, como cada miércoles a esta hora.

(Böll 1962/1981: 215, traducido por Moral Arroyo)

En Das Abenteuer eines Brotbeutels, también se neutraliza en zoológico (TM2) frente a Bahnhof Zoo (TO, TM1). En este caso, el segundo traductor cae en un error, que parece ser fruto de una laguna cultural, al convertir la estación de tren de Berlín Bahnhof Berlin Zoologischer Garten, conocida como Bahnhof Zoo, en un "zoológico", omitiendo de nuevo el nombre propio:

(10) Er nahm seinen Abschied, bekam ein Bild des Generals Gublanez geschenkt und gelangte auf Umwegen nach Berlin, und als er vom Bahnhof Zoo mit der Straßenbahn nach Spandau fuhr, [...].

(Böll 1962: 26)

(a) Pidió el retiro, le regalaron un retrato del general Gublanez, tras muchos rodeos llegó a Berlín, y cuando desde la estación Zoo el tranvía le llevaba a Spandau, [...].

(Böll 1962/1964: 27-28, traducido por Fontseré)

(b) Pidió el retiro, recibió un retrato del general Gublánez y llegó tras muchos rodeos a Berlín y, al tomar el tranvía en el zoológico para dirigirse a Spandau, [...].

(Böll 1962/1981: 18, traducido por Moral Arroyo)

Un ejemplo de glosa extratextual en el corpus se da en el nombre Götz von Berlichingen, en el relato Abenteuer eines Brotbeutels, que se conserva como en el TO en ambas traducciones. Sin embargo, el segundo traductor, siguiendo su tendencia a acercar el TO a los lectores, introduce una nota a pie de página:

(11) Stobski taten die Füße immer noch weh, sie taten ihm sehr weh, und er ließ Habke sein, setzte sich auf eine nasse Wiese, die nach Kuhdung roch, und dachte etwas, was auf polnisch ungefähr einer Übersetzung des Spruchs von Götz von Berlichingen gleichgekommen wäre.

(Böll 1962: 23) 
(a) A Stobski le seguían doliendo los pies, le dolían mucho, y dejó que Habke fuera por donde quisiera, se sentó en un prado húmedo que todavía olía a estiercol de vaca y pensó en algo que en polaco habría venido a ser una traducción de la frase de Götz von Berlichingen.

(Böll 1962/1964: 24, traducido por Fontseré)

(b) A Stobski le seguían doliendo los pies, le dolían los pies, le dolían muchísimo, se olvidó de Habke, se sentó en un prado húmedo que olía a estiércol de vaca y pensó lo que en polaco correspondía aproximada-mente al dicho de Götz von Berlichingen.

(Böll 1962/1981: 15, traducido por Moral Arroyo)

La nota a pie de página aclaratoria dice: "«Que te chupen el c...», frase de Götz von Berlichingen, en la obra dramática del mismo nombre de J. W. von Goethe.” (Böll 1962/1981: 15, traducido por Moral Arroyo). El lector de español que desconozca esta obra no puede comprender en el TM1 el pensamiento del protagonista del relato de Heinrich Böll.

Siguiendo esa tendencia de evitar el extrañamiento en el lector, el traductor del TM2 opta por traducir al castellano Siedlung Tannenidyll, en el relato Wie in schlechten Romanen (Böll 1962), como Urbanización Idílico Pinar, mientras que el primer traductor conserva parte del nombre propio en alemán: Polígono Tannenidyll.

(12) Frau Zumpen holte die Kognakflasche, schenkte ein, und nocht bevor ich etwas von dem Auftrag hatte sagen können, schob sie mir einen gelben Schnellhefter zu:»Siedlung Tannenidyll«las ich und blickte erschrocken auf Frau Zumpen, [...].

(Böll 1962: 119)

(a) Sacó la botella del coñac, nos sirvió, y antes de que yo pudiera hablar de la concesión me pasó una carpeta amarilla. Vi que decía "Polígono Tannenidyll" y miré asustado a la Zumpen [...].

(Böll 1962/1964: 190, traducido por Fontseré)

(b) La señora Zumpen buscó la botella de coñac, llenó las copas y antes de que yo pudiera hablar del encargo, me pasó un clasificador amarillo: «Urbanización idílico Pinar", leí y miré asustado a la señora Zumpen, [...].

(Böll 1962/1981: 215, traducido por Moral Arroyo)

Como se observa en el texto, el segundo traductor conserva el tratamiento de respeto hacia la señora Zumpen, siguiendo el original, mientras que el primer traductor, la denomina la Zumpen, introduciendo un matiz coloquial que no se justifica en su contexto y que sorprende al lector.

Una mención especial merecen los antropónimos que hacen referencia a personajes históricos. En estos casos, los traductores de los tres dobletes coinciden en reemplazarlos por las formas reconocidas en español, como se observa en el Anexo 1: Budda/Buda, Sokrates/Sócrates, Napoleon/Napoleón, Homer/Homero, Krösus/ Creso en Traumfährte y sus traducciones (Hesse); Aristoteles/Aristóteles en Tragisch y sus traducciones (Hesse); Kain/Caín, Judas Ischariot/Judas Iscariote en Vogel y sus traducciones (Hesse); heiliger Franziskus/San Francisco, Jesus Christus/Jesucristo en Das Abenteuer y sus traducciones (Böll); Judas Taddäus/Judas Tadeo en Daniel der Gerechte y sus traducciones (Böll). Coinciden, asimismo, en conservar como en el original los apellidos de personajes conocidos: Uhland, Schubert, Shakespeare en 
Traumfährte y sus traducciones (Hesse); Nietzsche, Kleist, Brentano, Hoffmann, Mörike, Stifter, Jean Paul, Armin, Eichendorff, Heine en Tragisch y sus traducciones (Hesse); Mozart en Vom Steppenwolf y sus traducciones (Hesse); Don Bosco en Daniel der Gerechte y sus traducciones (Böll). En el TM1 de Vom Steppenwolf (Hesse), el traductor prefiere Johann Wolfgang Goethe (TM1), a Johann Wolfgang von Goethe (TO, TM2).

Sin embargo, el nombre Jesús, refiriéndose al hijo de Dios, desaparece del TM1 de Vogel en el siguiente contexto:

(13) Gewiss, es gab Vollendete, es gab Menschgötter, es gab einen Budda, es gab einen Jesus, es gab einen Sokrates.

(Hesse 1945/1987: 344)

(a) Ha habido seres superiores, no puede negarse, hombres-dioses. Ha habido un Buda, un Sócrates.

(Hesse 1945/1950: 10, traducido por Pintó)

(b) Ciertamente existían los perfectos, los dioses humanos, había existido Buda, Jesús, Sócrates.

(Hesse 1945/1976: 27, traducido por Bofill)

En esta traducción del año 1950, la más antigua de las analizadas, la omisión puede venir motivada por la censura del franquismo en España. Podría considerarse un ataque a la Iglesia el identificar la figura de Jesús con un "hombre-dios" y compararlo con Buda y Sócrates.

Asimismo se adaptan los nombres propios de lugares con traducción reconocida, como Türkei (Dürrenmatt 1952/1986), traducido por Turquía en los TM1 y TM2; Indien (Hesse 1945/1987), traducido por India en los TM1 y TM2, y Köln (Böll 1962), traducido por Colonia en los TM1 y TM2.

Bajo la perspectiva de la técnica de traducción más empleada, el análisis cuantitativo del total de los dobletes desprende, como era de esperar, que la repetición es el procedimiento más utilizado por los traductores (66\%), seguida de la adaptación (21\%) y la traducción lingüística (10\%), mientras que el resto de técnicas apenas tiene representación (3\%: la glosa intratextual con un 1\%, la omisión 1\%, la neutralización y la naturalización $0,7 \%$ y la glosa extratextual [en concreto, la técnica de la nota a pie] $0,3 \%)$.

CUADRO 1

Porcentaje de nombres propios traducidos según la técnica empleada

\begin{tabular}{|l|c|}
\hline Técnica & Porcentaje \\
\hline Repetición & $66 \%$ \\
\hline Adaptación & $21 \%$ \\
\hline TL & $10 \%$ \\
\hline Otras & $3 \%$ \\
\hline
\end{tabular}

Si se le añade el parámetro de la tipología del nombre propio a este análisis cuantitativo, se reflejan los siguientes resultados en términos porcentuales para antropónimos (280) y topónimos (264): 
CUADRO 2

Porcentaje de nombres propios traducidos según tipología y técnica empleada

\begin{tabular}{|l|c|}
\hline \multicolumn{2}{|c|}{ Antropónimos } \\
\hline Técnica & Porcentaje \\
\hline Repetición & $75 \%$ \\
\hline Adaptación & $18 \%$ \\
\hline TL & $5 \%$ \\
\hline Otras & $2 \%$ \\
\hline
\end{tabular}

\begin{tabular}{|l|c|}
\hline \multicolumn{2}{|c|}{ Topónimos } \\
\hline Técnica & Porcentaje \\
\hline Repetición & $57 \%$ \\
\hline Adaptación & $25 \%$ \\
\hline TL & $14 \%$ \\
\hline Otras & $5 \%$ \\
\hline
\end{tabular}

En base a estos resultados se pone de relieve que la reproducción del nombre propio sin ningún cambio en las traducciones es mayor en el caso de los antropónimos y que, en los topónimos, la traducción lingüística adquiere una mayor importancia, debido fundamentalmente a la transparencia semántica que caracteriza algunos de ellos, como lo ilustran estos ejemplos: Bielersee (Dürrenmatt 1952/1986), traducido por lago de Biel en los TM1 y TM2; Bahnhofsplatz (Dürrenmatt 1952/1986), traducido por plaza de la estación en los TM1 y TM2. El resto de técnicas traductológicas apenas tiene representación en el corpus analizado.

\section{Conclusiones}

La primera reflexión que subyace de este análisis es que los nombres propios, al igual que cualquier otro elemento textual, sí se traducen. Como hemos sostenido a lo largo de este estudio, defendemos la traducibilidad teórica y práctica de los nombres propios, al considerar la traducción como un proceso en el que el traductor, en un momento dado, reflexiona y elige una opción traslatoria concreta, entre las que se encuentran la repetición y la adaptación. Tal y como señala Antoine Berman en su obra L'Épreuve de l'étranger (1984), trabajo con un ilustrativo título en el que el autor aborda la traducción de textos humanísticos con un enfoque de gran universalidad y profundidad filosófica, «la non-traduction d'un terme [vaut] comme un mode éminent de traduction» (Berman 1984: 302).

Siguiendo con el hilo conductor de esta primera reflexión, tras el análisis de los resultados concluimos destacando que la repetición es la técnica más empleada por los seis traductores en estas obras literarias dirigidas al público adulto, lo que corrobora nuestra hipótesis de partida. A continuación le siguen la adaptación, ya sea ortográfica o terminológica, y la traducción lingüística, mientras que el resto de técnicas apenas tiene representación en el caso de antropónimos y topónimos. Una mención especial merecen, sin embargo, los nombres propios que cuentan con un referente dotado de traducción prefijada, en estos casos, los seis traductores suelen coincidir en remplazarlos por las formas consagradas en español, las que marcan la tradición traductora.

En el corpus analizado, los nombres propios forman parte de obras literarias, por ello el traductor debe cuestionarse, en primer lugar, si se trata de nombres reales, o nombres ficticios, en segundo lugar, qué grado de transparencia tienen, así como también qué grado de caracterización poseen y, por último, su grado de popularidad. No sabemos hasta qué punto nuestros traductores se habrán planteado estas cuestiones, pues tras el análisis traductológico de los dobletes se desprende que los criterios de los traductores a la hora de traducir un nombre propio distan de ser homogéneos 
y que incluso en un mismo traductor se observan inconsecuencias en su tratamiento. No obstante, sí puede afirmarse que Juan José del Solar, el segundo traductor del relato de Friedrich Dürrenmatt, es el que en mayor medida, en palabras de Schleiermacher, «lässt den Schriftsteller möglichst in Ruhe, und bewegt den Leser ihm entgegen» [deja tranquilo al escritor y hace que el lector se acerque a él] (Schleiermacher 1818, citado en Störig 1963: 47). No nos extraña este proceder, tratándose de un traductor especializado en el autor suizo que hasta 1989, año en el que se publica esta traducción, había vertido ya al español once de sus obras. El conocimiento de la labor literaria del autor se refleja asimismo en el acierto en las soluciones traductológicas que propone $\mathrm{e}^{17}$.

Introduciendo la perspectiva diacrónica, se observa que es en la traducción más antigua de las analizadas, la primera de Hermann Hesse, realizada en 1950, en la que en mayor medida se advierte la tendencia a acercar el texto alemán a los lectores, evitando el efecto de extrañeza. De modo ilustrativo cabe destacar que en este primer traductor se percibe una tendencia a adaptar los antropónimos, muy especialmente los nombres de pila que comparten etimología con el español, algo que caracteriza su tratamiento en España desde los años veinte a principios de los sesenta, con una década y media más de transición (Franco Aixelá 2000: 261). De esta manera, los cuarenta y nueve años que separan la primera de las traducciones analizadas (1950) y la última (1989) vendrían a reflejar una evolución a lo largo de estas décadas en el tratamiento del nombre propio.

Asimismo se ha observado en el análisis que esta primera traducción de los relatos de Hesse se caracteriza por continuos despistes o errores. Hemos ilustrado la falta de cuidado traductor y editorial con algunos ejemplos: en las obras de Hesse, Schuster (Vogel [TO], TM2) se convierte en Sehuster (TM1); Justinus Kerner (Schwäbische Parodie [TO], TM2) es Instinus Kerner (TM1); Bopfingen (Vom Steppenwolf [TO], TM2) pasa a Boptingen (TM1); y Hohenstaufen (Vogel [TO]) aparece en el TM1 con las grafías Hoheustaufen y Hohenstaufen.

Desde una óptica temporal, este fenómeno, no encontrado en ninguna de las traducciones posteriores, podría indicar una evolución en la calidad de las traducciones en este sentido; en cualquier caso, lo que sí parece atisbarse es una mayor sensibilización hacia el proceso traductor, sin duda gracias al avance en la epistemología de la traducción, en parte por haber llegado como disciplina autónoma a las aulas universitarias, con las favorables consecuencias que esta mayor conciencia traductora conlleva para la calidad de la traducción.

Continuando con el enfoque diacrónico, ahora desde el plano de cada uno de los dobletes, las primeras traducciones realizadas tienden a hacer uso de la adaptación y la traducción lingüística en mayor proporción que las segundas, que emplean mayormente la repetición ${ }^{18}$. Estos resultados observados en cada uno de los dobletes coinciden con la evolución que constata Franco Aixelá en su estudio: mayor tendencia a la adaptación y traducción lingüística hasta los años sesenta, con un periodo de vacilación hasta la segunda mitad de los setenta (Franco Aixelá 2000: 258).

La perspectiva temporal hace especialmente interesante el análisis de dobletes de traducción al permitir estudiar el concepto de traducción que pueda tener una sociedad en un momento determinado. Sin lugar a duda, en el mundo de hoy, cada vez más globalizado y más intercultural, la traducción juega un papel fundamental como acercamiento entre culturas, pero siempre desde el respeto a la singularidad 
de cada una de ellas. Siguiendo esta reflexión, parece comprensible, como se desprende del análisis, que desde un enfoque diacrónico se observe una mayor "extranjerización" en las segundas traducciones, en palabras de Berman, traducción «aliénante», frente a las primeras traducciones en las que se evita en mayor medida el efecto de extrañeza del lector, traducción «ethnocentrique» (Berman1984: 201, 297; 2008: 48). Desde un enfoque global y universal de la traducción, se aboga, siguiendo a este autor, por una teoría de la traducción non ethnocentrique, por el respeto al otro, por el diálogo con las otras culturas. En líneas generales, el análisis vendría a reflejar esa evolución a la que alude Berman y que le lleva a afirmar que "la traduction moderne doit être dialogique" (Berman 1984: 287).

Por otra parte, consideramos que resulta especialmente interesante el estudio de diferentes traducciones de un mismo texto original, en primer lugar, porque el cotejo intralingual permite extraer deducciones sobre los propios traductores, su concepción del texto, sus principios de traducción, sus particularidades individuales de estilo, etc. En este sentido, se le atribuye al traductor un papel clave, al ser el responsable de articular dos situaciones de enunciación, evitando lo que Barbara Folkart denomina "le conflit des énonciations" (Folkart 1986: 251). Como se ha visto en el análisis, la omisión del término Jesús por el primer traductor del texto de Hesse ilustra la elección, en este caso ideológica, motivada por la censura de la dictadura franquista en España en 1950. El traductor evita con la omisión un posible ataque a la Iglesia, lo que ilustra que se traduce siempre a partir de "une certaine position dans l'espace socioculturel, temporel et géographique" (Folkart 1991: 11).

En segundo lugar, y desde una óptica interlingual, la comparación entre dobletes de traducción puede mostrar asimismo puntos de contrastividad entre las lenguas analizadas y poner de relieve dónde radican las dificultades a la hora de traducir un texto entre ese par de lenguas, con las consecuentes ventajas para la traducción y su didáctica. Es precisamente en el nombre propio, uno de esos lugares en los que de manera más evidente se muestra la alteridad del texto original y del texto meta.

Mais le nom propre constitue également le lieu où se manifeste avec le plus d'évidence l'alterité radicale du texte source et du texte cible: lieu de démarquage traversé de toutes les tensions entre deux textes et deux matrices culturelles, il atteste ce conflit d'énonciations qui est au cœur même de la traduction. (Folkart 1986: 251)

Por ello, queremos destacar en este estudio la importancia que tiene el reflexionar sobre el tratamiento del nombre propio en este contexto traductológico, por el avance que esto supone también para la didáctica de la traducción ${ }^{19}$.

En esta misma línea, deseamos llamar la atención sobre el hecho de que en España sigan siendo escasos los trabajos que se hayan dedicado con profundidad al tema (Moya 2000; Franco Aixelá 2000; y las tesis inéditas de Barros Ochoa 1995 y Adrada Rafael 2009), en contraposición con lo que sucede en otros países, fundamentalmente en Alemania y en el contexto francófono.

Incidimos, finalmente, en la idea de que el acercamiento al análisis del nombre propio ha de hacerse de modo holístico, abandonando la dicotomía traducibilidad/ intraducibilidad y renunciando a un enfoque estrictamente lingüístico. El nombre propio, como cualquier otro elemento del texto original, es considerado por el traductor en su toma de decisiones, está presente en el proceso cognitivo de reenunciación del texto. De tal manera que, desde esta triple óptica (cognitiva, textual 
y comunicativa), resulta palmaria la importancia de la posición del traductor a la hora de traducir un nombre propio, por las implicaciones que esto conlleva para el propio texto $y$, consiguientemente, para el efecto comunicativo de los futuros lectores de la traducción.

\section{NOTAS}

1. Véase el extenso e interesante trabajo de J.-L. Vaxelaire (2005) en el que presenta un exhaustivo análisis histórico y epistemológico del nombre propio.

2. Diccionario de la Real Academia (1992): Madrid: Espasa-Calpe.

3. Diccionario de la Real Academia (2001): Madrid: Espasa-Calpe.

4. Diccionario de la Real Academia. Consultado el 9 de agosto 2013, <www.rae.es>.

5. «Die semantischen Besonderheiten der Namen gegenüber den Appellativa bedeuten eine schwierige linguistische Problematik und darüber hinaus auch ein philosophisches Problem» (Jäger y Jäger 1968: 131). [Las peculiaridades semánticas de los nombres propios frente a los nombres comunes suponen una problemática lingüística compleja y, además, un problema filosófico].

6. DürRenMatT, Friedrich (1952/1986): Der Richter und sein Henker. Zürich: Diogenes.

7. Hesse, Hermann (1945/1987): Traumfährte. Frankfurt a. M: Suhrkamp. Suhrkamp Taschenbuch 1600, Gesammelte Werke in zwölf Bänden. Los relatos analizados para este estudio son: Traumfährte, Tragisch, Kindheit des Zauberers, Schwäbische Parodie, Vom Steppenwolf, König Yu y Vogel.

8. BöLL, Heinrich (1962): Erzählungen, Hörspiele, Aufsätze. Köln/Berlin: Kiepenheuer \& Witsch. Los relatos analizados son: Das Abenteuer, Abenteuer eines Brotbeutels, Der Zwerg und die Puppe, So ward Abend und Morgen, Unberechenbare Gäste, Daniel der Gerechte, Wie in schlechten Romanen y Eine Kiste für Kop.

9. Dürrenmatt, Friedrich (1952/1989): El juez y su verdugo. (Traducido por José Del Solar) Barcelona: Tusquets Editores.

10. Dürrenmatt, Friedrich (1952/1970): El juez y su verdugo. (Traducido por Inge S. DE LuQue [1962]) Barcelona: Planeta.

11. Hesse, Hermann (1945/1976): Rastro de un sueño. (Traducido por Mireia Bofill) Barcelona: Planeta.

12. Hesse, Hermann (1945/1950): Ensueños. (Traducido por Alfonso Pintó) Barcelona: Caralt.

13. El nombre Schuster se repite varias veces en el relato y en todas ellas como Sehuster (Hesse 1945/1950: 138; 142; 144; 148).

14. Unas líneas más adelante aparece, sin embargo, el término correcto Hohenstaufen, otro ejemplo de falta de cuidado en la redacción de la traducción (Hesse 1945/1950: 153, traducido por Pintó).

15. BöLL, Heinrich (1964): La aventura y otros relatos. (Traducido por Margarita FonTSERÉ) Barcelona: Seix Barral.

16. BölL, Heinrich (1981): La aventura y otros relatos. (Traducido por José Moral Arroyo) Barcelona: Bruguera.

17. Precisamente su actividad traductora en lengua alemana, en la que se incluye autores de la talla de Elias Canetti, Bertold Brecht, Tomas Mann y Franz Kafka, ha sido laureada con varios galardones, entre ellos, el Premio de Traducción del Ministerio de Asuntos Exteriores de la República Federal Alemana (1985, véase Benítez 1992: 204) y el Premio Nacional a la Obra de un Traductor (2004), premio nacional español creado para reconocer la trayectoria de traductores literarios. Véase Oвiols, Isabel (12 de noviembre de 2004): Juan José del Solar, Nacional de Traducción por toda su obra. El País. Consultado el 9 de agosto de 2013 <http://elpais.com/diario/2004/11/12/cultura/ 1100214005_850215.html>.

18. A excepción del doblete de Heinrich Böll que, como ya se ha comentado, precisamente la distancia entre ambas traducciones, era menor y es muy poco significativo el número de divergencias entre los dos traductores, coincidiendo en el $85 \%$ de las soluciones.

19. En las clases de traducción estamos acostumbrados a que una de las primeras dudas que surgen a los estudiantes cuando se enfrentan a un encargo de traducción sea: ¿Qué hacemos con los nombres propios? ¿Los debemos traducir? Nuestra experiencia nos muestra que lo importante es que el traductor considere el encargo de traducción en su conjunto, cada caso en concreto, cada autor, cada obra, cada nombre propio y actúe en consecuencia, desarrollando con ello una competencia pragmática que será fundamental para el buen éxito del encargo de traducción. 


\section{REFERENCIAS BIBLIOGRÁFICAS}

Adrada Rafael, Cristina (2009): Antroponimia y connotación: la traducción al español de los nombres de persona en la obra de Molière. Tesis de doctorado no publicada. Valladolid: Universidad de Valladolid.

Allerton, David J. (1987): The linguistic and sociolinguistic status of proper names. What Are They, And Who Do They Belong To? Journal of Pragmatics. 11:61-92.

Ballard, Michel (2001): Le nom propre en traduction. Paris-Gap: Ophris.

BALLARD, Michel (2011): Épistémologie du nom propre en traduction. In: Georgiana LunguBadea y Alinea Pelea, eds. (In)Traductibilité des noms propres. Translationes. 3:33-47.

Barros OchoA, María José (1994): La traducción del nombre propio inglés-español: teoría y práctica. Tesis de doctorado no publicada. León: Universidad de León.

Berman, Antoine (1984): L'épreuve de l'étranger. Culture et traduction dans l'Allemagne romantique. Paris: Gallimard.

Berman, Antoine (2008): L'âge de la traduction: «La tâche du traducteur» de Walter Benjamin, un commentaire. París: Presses Universitaires de Vincennes.

Benítez, Esther (1992): Diccionario de traductores. Madrid: Fundación Germán Sánchez Ruipérez.

BrÉAL, Michel (1924/1976): Essai de sémantique. Science des significations. Genève: Slatkine Reprints.

Delisle, Jean (1984): L'analyse du discours comme méthode de traduction. Initiation à la traduction des textes pragmatiques anglais. Ottawa: Université d'Ottawa.

Donato (IV d. C.): Ars grammatica. In: Heinrich KeIL eds. Grammatici Latini. Vol. IV. Hildesheim: Georg Olms, 1981, 353-402.

EICHLER, Ernst (1995): Namenforschung: Überblick, Geschichte, Richtungen, Institutionen. In: Ernst EICHLER, ed. Namenforschung. Ein internationales Handbuch zur Onomastik. Vol. 1. Handbuch zur Sprach- und Kommunikationswissenschaft. Berlín/Nueva York: Walter de Gruyter, 1-7.

Fernández Leborans, María Jesús (1999): El nombre propio. In: Ignacio Bosque y Violeta Demonte, eds. Gramática descriptiva de la lengua española. Vol. 1. Madrid: Espasa, 77-128.

Fleischer, Wolfgang (1964): Zum Verhältnis von Name und Appellativum im Deutschen. Wissenschaftliche Zeitschrift der Karl-Marx-Universität Leipzig. 13:369-378.

FLeISCHER, Wolfgang (1973a): Onomastik und Stilistik. In: Namenkundliche Informationen, Heft 22/Mai 1973. Beiträge zum 20. Jahrestag der Namensgebung der Karl-Marx-Universität. Leipzig: Universität Leipzig, 5-12.

Fleischer, Wolfgang (1973b): Variationen von Eigennamen. In: Ernst Eichler, Wolfgang Fleischer, Rudolf Grosse et al., eds. Der Name in Sprache und Gesellschaft. Beiträge zur Theorie der Onomastik. Vol. 27. Berlín: Akademie-Verlag, 52-63.

FolKarT, Barbara (1986): Traduction et remotivation onomastique. Meta. 31(3):233-252.

FolKART, Barbara (1991): Le conflit des énonciations. Traduction et discours rapporté. Québec: Les Éditions Balzac.

FolKART, Barbara (2007): Second Finding. A poetics of translation. Ottawa: University of Ottawa Press.

Franco AiXelá, Javier (2000): La traducción condicionada de los nombres propios. Salamanca: Almar.

Funke, Otto (1925): Zur Definition des Begriffs 'Eigenname’. In: Wolfgang Keller, ed. Probleme der englischen Sprache und Kultur. Festschrift J. Hoops zum 60. Geburtstag. Heidelberg: Heidelberg, C. Winter, 72-79.

García Yebra, Valentín (1989): En torno a la traducción. Madrid: Gredos.

GARY-Prieur, Marie-Noëlle (1991): Le nom propre constitue-t-il une catégorie linguistique? Langue française. 92:4-26.

GerhardT, Dietrich (1949/1950): Über die Stellung der Namen im lexikalischen System. Beiträge zur Namenforschung. 1:1-24.

GLÄSER, Rosemarie (1976): Zur Übersetzbarkeit von Eigennamen. Linguistische Arbeitsberichte. 13: 12-25. 
Grass, Thierry (2002): Quoi! Vous voulez traduire «Goethe»? Essai sur la traduction des noms propres allemand-français. Bern: Peter Lang.

Grass, Thierry, Humbley, John y Vaxelaire, Jean-Louis, eds. (2006): Meta. 51(4).

Gutsснміdт, Karl (1980): Bemerkungen zur Wiedergabe von Eigennamen beim Übersetzen. Studia Onomastica I: 47-54 (Beiheft zu Namenkundliche Informationen, 2).

Hellfritzsch, Volkmar (1973): Zum Problem der stilistischen Funktion von Namen. In: Ernst Eichler, Wolfgang Fleischer, Rudolf Grosse et al., eds. Der Name in Sprache und Gesellschaft. Beiträge zur Theorie der Onomastik. Vol. 27. Berlín: Akademie-Verlag, 64-73.

JäGER, Gert y JÄGER, Sonja (1968): Zum Problem der Namen beim Übersetzen aus dem Deutschen ins Spanische. Fremdsprachen. 2:131-134.

Jespersen, Otto (1924/1992): The Philosophy of Grammar. Chicago/Londres: The University of Chicago Press.

KALVERKÄMPER, Hartwig (1978): Textlinguistik der Eigennamen. Stuttgart: Klett-Cotta.

KALVERKÄMPER, Hartwig (1995): Namen im Sprachaustausch: Namenübersetzung. In: Ernst EICHLER, ed. Namenforschung. Ein internationales Handbuch zur Onomastik. Vol. 2. Handbuch zur Sprach- und Kommunikationswissenschaft. Berlín/Nueva York: Walter de Gruyter, 1018-1025.

Lungu-Badea, Georgiana y Pelea, Alinea, eds. (2011): Translationes. Vol. 3. (In)Traductibilité des noms propres. Timisoara: Centre d'Études de la Traduction de l'Université de l'Ouest.

LUNGU-BADEA, Georgiana (2011): La traduction (im)propre du nom propre littéraire. In: Georgiana Lungu-Badea y Alinea Pelea, eds. (In)Traductibilité des noms propres. Translationes. 3:65-79.

MAŃCZAK, Witold (1969): Différence entre nom propre et nom commun. In: Abhandlungen des X. Kongreßes für Namenforschung. Vol. 2. Wien:Akademischer Verlag, 285-291.

MiLL, John Stuart (1875): A System of Logic, ratiocinative and inductive. $9^{\text {th }}$ ed. Vol. 1. London: Longmans, Green, Reader, and Dyer.

Molino, Jean (1982): Le nom propre dans la langue. Langages. 66:5-20.

Morala, José R. (1986): El nombre propio, ¿objeto de estudio interdisciplinar? Contextos IV(8): 49-61..

MoyA, Virgilio (2000): La traducción de los nombres propios. Madrid: Cátedra.

Neubert, Albrecht (1973): Name und Übersetzung. In: Ernst Eichler, Wolfgang Fleischer, Rudolf Grosse et al., eds. Der Name in Sprache und Gesellschaft. Beiträge zur Theorie der Onomastik. Vol.27. Berlín: Akademie-Verlag, 74-79.

Newmark, Peter (1988a): A Textbook of Translation. Nueva York: Phoenix Elt.

Newmark, Peter (1988b): Approaches to Translation. Londres/Nueva York/Toronto: Prentice Hall.

Nord, Christiane (2003): Proper Names in Translations for Children: Alice in Wonderland as a Case in Point. Meta. 48(1-2):182-196.

Рвовsт, Lilia (1984): Regelmäßigkeiten und Besonderheiten der Wiedergabe von Eigennamen bei der Übersetzung aus dem Deutschen in das Russische. Vol. 1. Berlín [s. i].

Pulgram, Ernst (1954): Theory of names. Beiträge zur Namenforschung. 5:149-196.

Schleiermacher, Friedrich (1818/1963): Über die verschiedenen Methoden des Übersetzens. In: Hans Joachim STörIG, ed. Das Problem des Übersetzens. Wege der Forschung. Darmstadt: Wissenschaftliche Buchgesellschaft.

Sorensen, Holger Steen (1963): The meaning of Proper Names. Kopenhagen: Gad.

STÖRIG, Hans, ed. (1963): Das Problem des Übersetzens. Wege der Forschung. Darmstadt: Wissenschaftliche Buchgesellschaft.

TORre, Esteban (1994): Teoría de la traducción literaria. Madrid: Síntesis.

VAXelaire, Jean-Louis (2005): Les noms propres. Une analyse lexicologique et historique. París: Champion.

Vaxelaire, Jean-Louis (2011): De Mons à Bergen. De l'intraduisibilité des noms propres. In: Georgiana Lungu-Badea y Alinea Pelea, eds. (In)Traductibilité des noms propres. Translationes. 3:13-28. 
Venuti, Lawrence (1995). The Translator's Invisibility. Londres/Nueva York: Routledge.

Venuti, Lawrence (1998). Strategies of translation. In: Mona BAKer, ed. Routledge encyclopedia of translation studies. Londres/ Nueva York: Routledge, 240-244.

Zabeen, Farhang (1968): What is in a Name? An Inquiry into the Semantics and Pragmatics of Proper Names. The Hague: Nijhoff.

Zimmer, Rudolf (1981): Probleme der Übersetzung formbetonter Sprache. Beihefte zur Zeitschrift für romanische Philologie, 181. Tübingen: Niemeyer.

\section{ANEXOS}

Anexo 1 Antropónimos que hacen referencia a personajes históricos

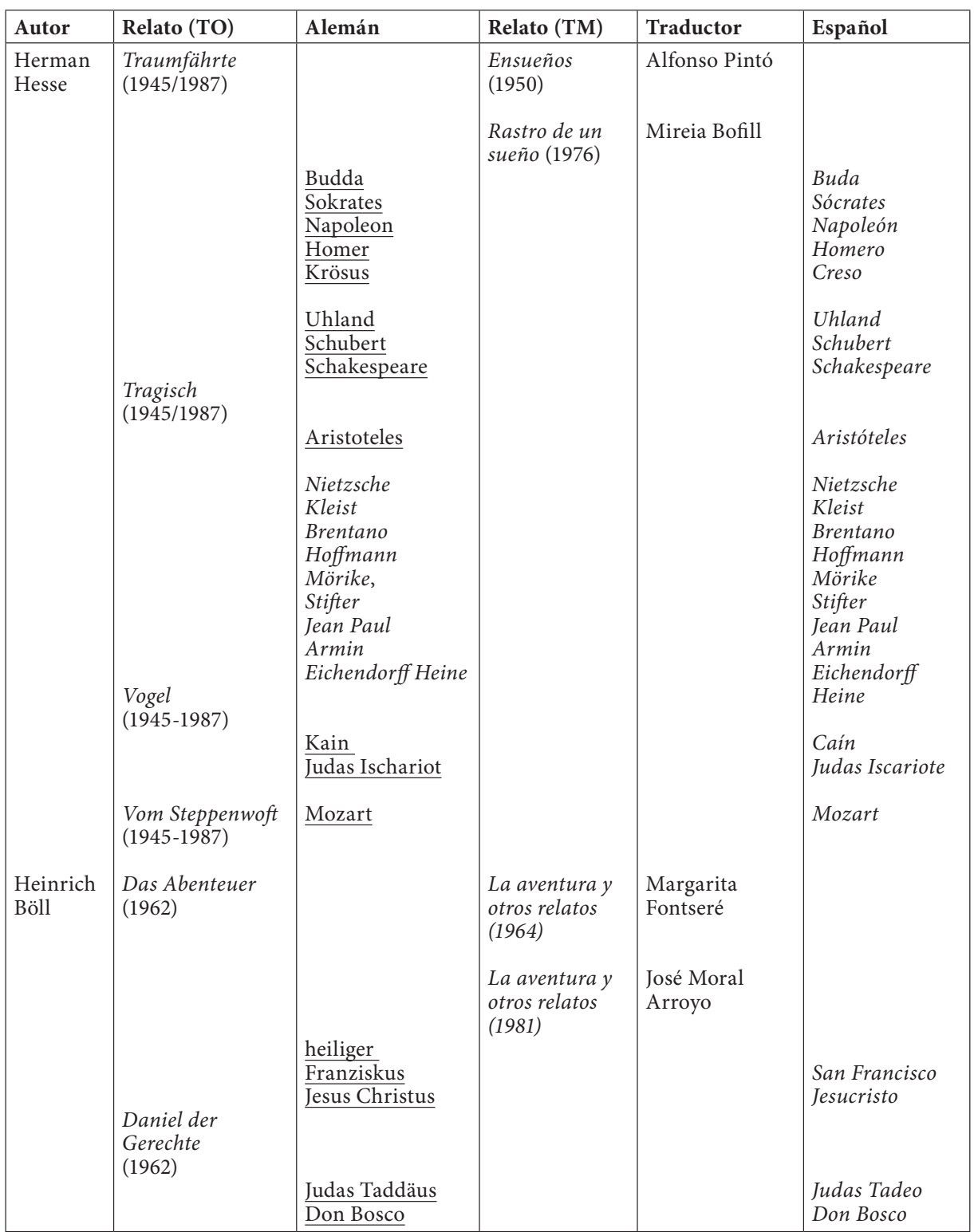

